\title{
Neural Circuit Reconfiguration by Social Status
}

\author{
Fadi A. Issa, ${ }^{1,3}$ Joanne Drummond, ${ }^{1}$ Daniel Cattaert, ${ }^{3}$ and Donald H. Edwards ${ }^{2}$ \\ ${ }^{1}$ Department of Biology and ${ }^{2}$ Neuroscience Institute, Georgia State University, Atlanta, Georgia, 30303, and ${ }^{3}$ Centre de Neurosciences Intégratives et \\ Cognitives, CNRS-UMR 5228, Universités Bordeaux 1 and Bordeaux 2, Bat B2-Biologie Animale, Avenue des Facultés, 33405 TALENCE cedex, France
}

The social rank of an animal is distinguished by its behavior relative to others in its community. Although social-status-dependent differences in behavior must arise because of differences in neural function, status-dependent differences in the underlying neural circuitry have only begun to be described. We report that dominant and subordinate crayfish differ in their behavioral orienting response to an unexpected unilateral touch, and that these differences correlate with functional differences in local neural circuits that mediate the responses. The behavioral differences correlate with simultaneously recorded differences in leg depressor muscle EMGs and with differences in the responses of depressor motor neurons recorded in reduced, in vitro preparations from the same animals. The responses of local serotonergic interneurons to unilateral stimuli displayed the same status-dependent differences as the depressor motor neurons. These results indicate that the circuits and their intrinsic serotonergic modulatory components are configured differently according to social status, and that these differences do not depend on a continuous descending signal from higher centers.

\section{Introduction}

Differences in social status among animals arise during competition for resources as winners display assertive behaviors and are recognized as dominant, while losers display submissive behaviors and are recognized as subordinate (Wilson, 1975; Herberholz et al., 2007). These behavioral differences result from changes in the nervous system induced by social experience. Social status affects neurogenesis in rodents (Kozorovitskiy and Gould, 2004) and crayfish (Song et al., 2007), neuronal size in fish (White et al., 2002), brain morphology in wasps (O'Donnell et al., 2007) and naked mole rats (Holmes et al., 2007), and cell receptor populations in crayfish (Spitzer et al., 2005) and fish (Burmeister et al., 2007). Social status also affects the serotonergic neuromodulation of synaptic responses in both crayfish (Yeh et al., 1996, 1997) and fish (Whitaker et al., 2011), and the excitability of neural circuits that produce different behaviors (Krasne et al., 1997; Herberholz et al., 2001; Neumeister et al., 2010). It remains unclear, however, how neural circuits are altered to produce status-dependent behavioral responses.

We report that in crayfish, local neural circuits that mediate responses to an unexpected touch are reconfigured according to

Received Nov. 9, 2011; revised Feb. 15, 2012; accepted March 6, 2012.

Author contributions: F.A.I., J.D., D.C., and D.H.E. designed research; F.A.I., J.D., D.C., and D.H.E. performed research; D.C. contributed unpublished reagents/analytic tools; F.A.I., J.D., D.C., and D.H.E. analyzed data; F.A.I. and D.H.E. wrote the paper.

This work was supported by National Science Foundation Research Grants 0135162 and 0641326 (to D.H.E.) and the Company of Biologists Travel Fellowship and the Brains and Behavior Fellowship (to F.A.I).. We thank Mr. Konstantin Mokhov, Laurel Johnston, Dr. Nadja Spitzer, and Dr. Brian Antonsen for technical assistance. We thank Drs. Paul Katz and Ronald Calabrese for their many helpful suggestions and comments regarding this project.

Correspondence should be addressed to Dr. Donald H. Edwards, Neuroscience Institute, Georgia State University, 850 Petit Science Center, 111 Jesse Hill Jr. Dr. SE, Atlanta, GA 30303. E-mail: dedwards@gsu.edu.

F. A. Issa's present address: Brain Research Institute, David Geffen School of Medicine, UCLA, 650 Charles Young Drive South, Box 915751, Room 53-231 CHS, Los Angeles, CA 90095-1751.

J. Drummond's present address: GlaxoSmithKline, 18095 Melbourne, Victoria 8003, Australia.

D. Cattaert's present address: Institut de Neurosciences Cognitives et Intégratives d'Aquitaine, Avenue des Facultés, 33405 TALENCE cedex, France.

DOI:10.1523/JNEUROSCI.5668-11.2012

Copyright $\odot 2012$ the authors $\quad 0270-6474 / 12 / 325638-08 \$ 15.00 / 0$ the animal's social status to produce different, status-dependent behavioral responses. Moreover, the reconfiguration of local circuits remains after isolation from higher centers, indicating that once altered by a change in the animal's status, the circuit configuration does not require a continuous hormonal or neural input to persist.

\section{Materials and Methods}

Animal maintenance. Crayfish [Procambarus clarkii (Girard), 8-10 cm] were bought from a commercial supplier (Atchafalaya Biological Supply) and housed individually in $5 \mathrm{~L}$ tanks, each containing dechlorinated water a filter, and an air-stone for water oxygenation. The water was changed with fresh water once every 2 weeks. The animals were isolated from each other physically, visually, and chemically for a minimum period of 1 month and were fed shrimp pellets twice weekly.

Pair formation. Dominance was established by pairing two previously isolated male crayfish. One day before the pairing, the animals were marked on the thorax with a permanent marker for identification. The following day, the animals were removed from their isolated tanks and placed in the testing tank. The testing tank was divided into two chambers with an opaque divider preventing interaction between the two animals. The animals were given 15 min to acclimate to the new environment before the divider was removed, freeing them to interact with each other. The agonistic interactions (attacks, approaches, escape tailflips, offensive tailflips, and retreats) between the two animals were recorded for the first $30 \mathrm{~min}$ of interactions and on every subsequent day. The animals were free to interact at all times during the pairing period, which lasted a minimum of 2 weeks and up to 1 month. Dominance between the paired animals was usually established within the first 15 min of interactions on day one and was rarely reversed. Dominance was determined based on the total number of aggressive and submissive behaviors an individual animal performed (for details, see Issa et al., 1999).

In vivo recordings. At the end of either the isolation or pairing periods, an animal was selected randomly for electrode implantation. The animal was chilled in ice water for 10 min to decrease its mobility, then placed ventral side up in a Petri dish and immobilized. A small incision was made in the posterior-ventral side of the coxopodite segment of the left fifth leg using a sharp, heated micropin. One pair of silver wire electrodes $(0.014 \mathrm{~cm} ; \mathrm{A}-\mathrm{M}$ Systems $)$ insulated with Teflon was inserted through the 


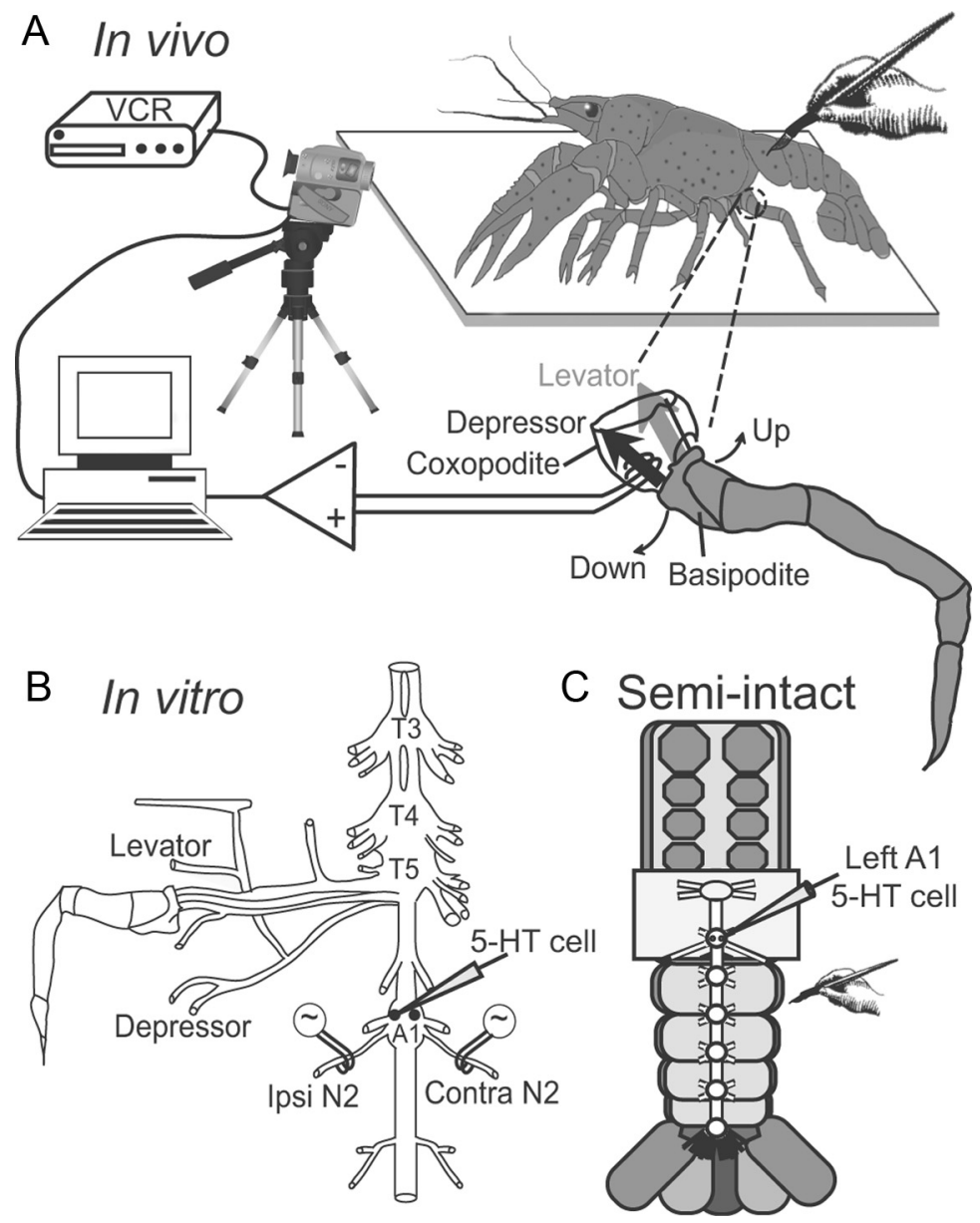

Figure 1. In vivo, in vitro, and semi-intact preparations. $A$, In vivo experiments. Potentials from the depressor nerve and muscle of the fifth walking leg were recorded by a bipolar pair of electrodes implanted around the nerve adjacent to the muscle. The animal's behavior was videotaped together with the reversed image of the electrical responses reflected from a mirror (not shown) in the viewing plane of the video camera. The behavioral and neuromuscular signals were recorded as the animal responded to an unexpected touch of the fringe hairs on one side of the first abdominal segment. $\boldsymbol{B}$, In vitro experiments. The animals of $\boldsymbol{A}$ were killed and the adjacent thoracic and abdominal ganglia were removed to a saline-filled dish, together with one or both fifth walking legs. Recording electrodes (not shown) were placed on left and right $T 5$ leg depressor nerves, and stimulating electrodes were placed on the left and right $\mathrm{N} 2$ of the A1. The soma of one A1 5-HT neuron was impaled with a microelectrode. Each sensory A1 N2 was electrically stimulated to mimic the brush stimulus delivered in vivo. C, Semi-intact preparations from dominant, subordinate, and isolate crayfish included the thorax and abdomen pinned ventral side up in saline. Ganglion A1 was exposed on a platform with its peripheral nerves and interganglionic connectives intact. An A15-HT cell was impaled and its responses to touch of the segmental fringe hairs were recorded.

hole and into the depressor muscle (Fig. 1). The electrodes were held in place with tissue adhesive (Vetbond; $3 \mathrm{M}$ ) applied to the leg cuticle. After surgery, the animal was placed in the testing tank alone for $24 \mathrm{~h}$ for recovery. The animal was then tested under dim red light to determine its behavioral response to an unexpected unilateral touch. The fringe hairs that project from the rostral abdominal pleural plate were brushed with a paintbrush that had a pair of fine wire electrodes attached parallel to the bristles. The paintbrush electrodes provided a reliable measure of the contact time of the brush with the animal because of the sudden change in resistance between the electrodes when contact was made. The animals were brushed randomly on either the left or the right side approximately every 10 min with brush duration of $\sim 1 \mathrm{~s}$.

A Canon digital camera (model 3CCD) captured video frames in a computer that simultaneously captured the depressor motor responses from the implanted electrodes and the brush stimulus-electrode responses. The behavior was also recorded on a standard videotape to ensure that no video frames were dropped during digital acquisition (Fig. 1). The depressor motor responses and animal's behavioral responses were time-locked and digitized using Spike2 (Cambridge Electronic Design).
In vitro electrophysiological recordings. Before dissection, an animal was chilled in icewater for $\sim 20 \mathrm{~min}$. Then it was decapitated and the thorax and abdomen were pinned dorsal side-up. A section of the ventral nerve cord containing ganglia from the second thoracic (T2) to first abdominal (A1) segments was removed while keeping the nerves from both fifth legs attached to the nerve cord (Fig. $1 B$ ). The nerve cord was pinned ventral side up at A1 ganglion for later intracellular recording from the ventrally located 5-HT cell body. Dissection and experiments were performed in physiological saline composed of the following (in $\mathrm{mm}$ ): $202 \mathrm{NaCl}, 5.37 \mathrm{KCl}, 13.53$ $\mathrm{CaCl}$, 2.6 MgCl2, 2.4 HEPES, 2.0 glucose, $\mathrm{pH}$ 7.4. Extracellular pin electrodes were placed on the second nerves at A1 for sensory stimulation. The extracellular signals were amplified by Grass amplifiers and then digitized (Spike2).

The soma of the A1 5-HT cell was impaled with a recording microelectrode filled with either $10 \%$ dextran rhodamine that was diluted in $2 \mathrm{M}$ potassium acetate or $5 \%$ Lucifer yellow with a resistance ranging from 35 to $45 \mathrm{M} \Omega$. The signals were amplified using Axon 2B amplifier (Molecular Devices) and then digitized for analysis and storage.

Data analysis. Spikes recorded from the depressor motor nerves and A1 5-HT cell were counted over the period that included the touch (in vivo preparations) or sensory nerve (in vitro preparations) stimulus. The counts from the first trial from each preparation were converted to firing frequency (spikes/s), and the frequencies from the corresponding times of trials from all animals were averaged. A two-tailed Wilcoxon matched pairs test was used to identify differences between the responses to ipsilateral and contralateral stimuli recorded at corresponding times relative to the stimulus. Other statistical tests were as described in the text.

5-HT immunohistochemistry. The immunocytochemical and histological procedures followed are described by Antonsen and Edwards (2003). Dehydrated preparations were cleared and mounted in methyl salicylate for laser scanning confocal microscopy (Zeiss

\section{LSM 510).}

Simulations. The circuit model was built and the simulations were run with AnimatLab, a neuromechanical simulator program available without charge at www.AnimatLab.com. The model neurons were singlecompartment, integrate-and-fire neurons, with a $1 \mathrm{M} \Omega$ input resistance, 5 $\mathrm{ms}$ time constant, $-60 \mathrm{mV}$ resting membrane potential, and $0.1 \mathrm{mV}$ membrane potential noise, and an initial firing threshold that differed according to the neuron (Fig. $5 \mathrm{~A}$ ). The rising phase of a spike was simulated as a voltage jump from the current membrane potential to $0 \mathrm{mV}$ in one integration time step $(0.2 \mathrm{~ms})$. A $1 \mathrm{mS}$ after-hyperpolarizing conductance immediately turned on and decayed exponentially thereafter with a time constant of $3 \mathrm{~ms}$. The corresponding current was determined by the product of this conductance and the difference between the current membrane potential and an equilibrium potential of $-70 \mathrm{mV}$. Synapses were simulated by an instantaneous increase in postsynaptic conductance that decayed with a single timeconstant. For excitatory synapses, the postsynaptic time constant was $10 \mathrm{~ms}$ and the reversal potential was $-10 \mathrm{mV}$; for inhibitory synapses, the corresponding values were $15 \mathrm{~ms}$ and $-70 \mathrm{mV}$, respectively. The postsynaptic 
A Isolate Contralateral response

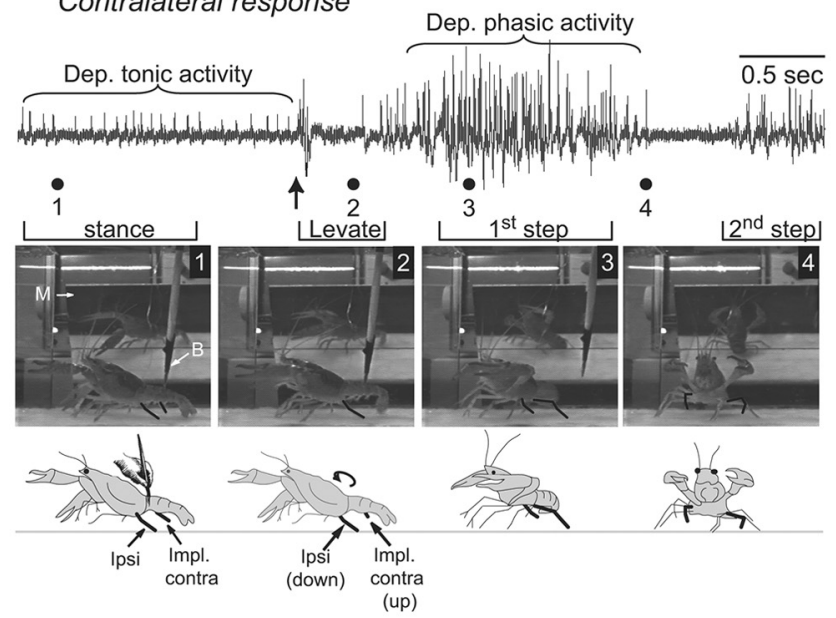

B Dominant

Ipsilateral response
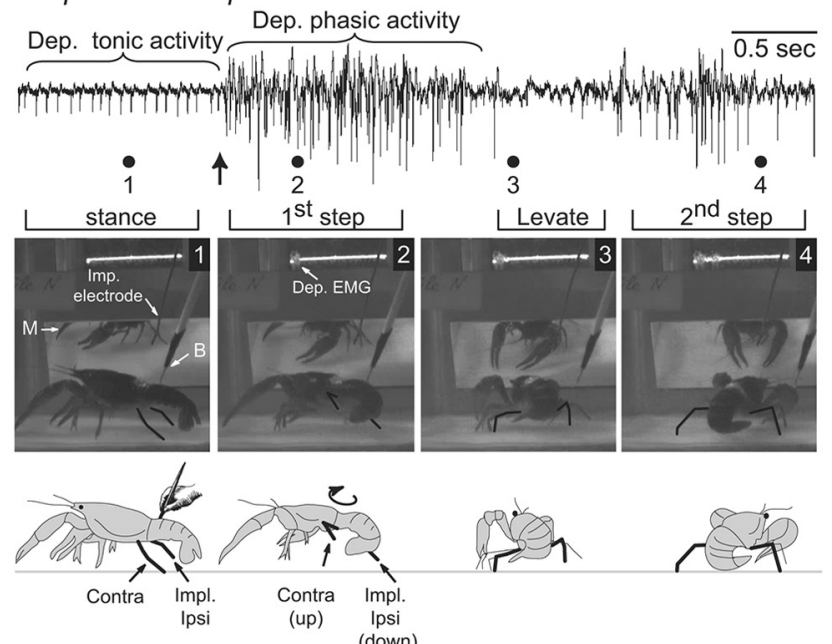

C Subordinate

Ipsilateral response

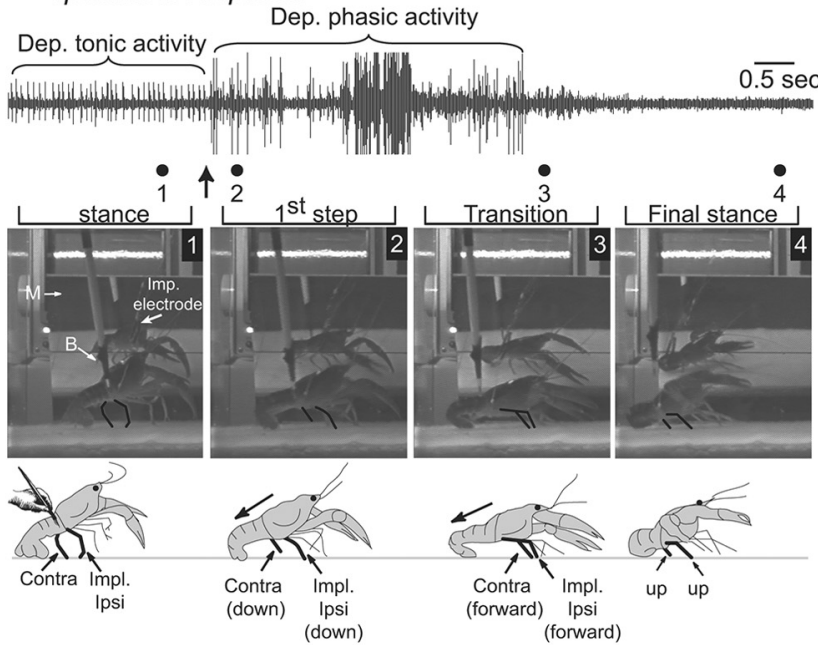

\section{D subordinate} Ipsilateral response

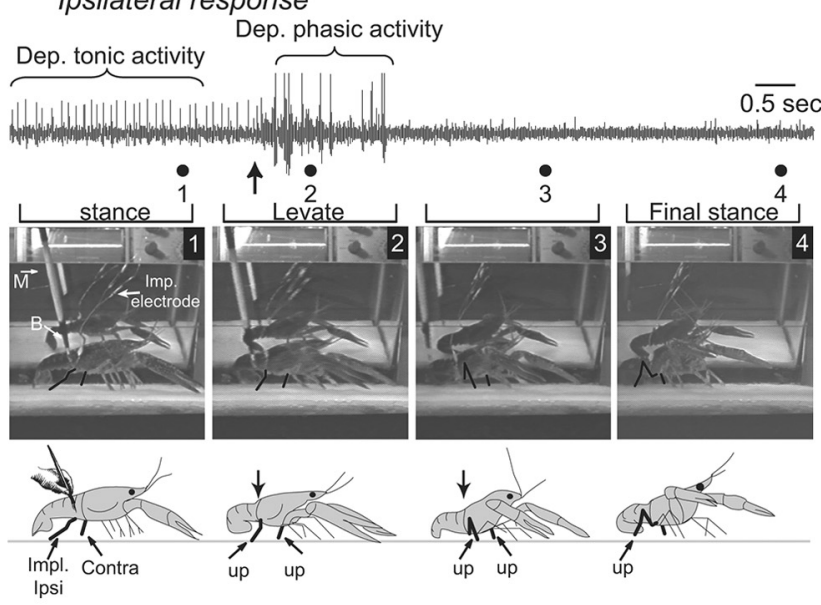

Figure2. Neuromuscular and behavioral responses of freely behaving socially isolated, dominant, and subordinate crayfish to an unexpected unilateral touch stimulus. The trace at the top of each panel shows the depressor neuromuscular activity recorded by the implanted fifth leg electrode in response to touch (upward arrow) of the abdominal fringe hairs. The numbered dots below each trace correspond to the time at which correspondingly numbered video frames were captured. The labeled brackets above the video frames indicate the periods of the electrical recording when the leg position was similar to that shown in the video frame. Video frames illustrate the animal's behavioral response to the brush stimulus. At the top of each video frame, the reflected oscilloscope screen was videotaped along with the behavior. Drawings illustrate the animal's posture and leg position in the video frame. Arrows on animals illustrate the rotation of body movements in response to the stimulus. M, Mirror; B, brush. A, Responses of a social isolate to touch to the left side of the abdomen; EMGs were recorded from right depressors. Turning occurs by depressing the left leg (Ipsi) while lifting the implanted right leg (Impl. contra). $\boldsymbol{B}$, Responses of a dominant to touch of the right side of the abdomen; EMGs were recorded from right depressors. Depressor muscle activation was displayed on the oscilloscope (Dep. EMG). C, Push response and right leg depressor EMGs of a subordinate to touch of the right side of the abdomen. $D$, Drop response and right leg depressor EMGs of a subordinate to touch of the right side of the abdomen.

conductances varied according to the synaptic connection (Fig. 6A). The model circuits are available at www.AnimatLab.com.

\section{Results}

Crayfish form long-lasting and stable social hierarchies in which socially subordinate animals (subordinates) give way to social dominants (dominants) in contests over resources (Copp, 1986; Edwards et al., 2003; Herberholz et al., 2007) and display other consistent differences in behavior, including the response to an unexpected unilateral touch (Rossi-Durand, 1993; Song et al., 2006). Simultaneous video and in vivo depressor EMG recordings of the responses of freely behaving crayfish to an unexpected touch to one side of the rostral abdomen revealed statusdependent differences in the behavioral and neuromuscular responses (Fig. 1). Socially isolated crayfish (isolates; Fig. 2A) and dominants (Fig. $2 B$ ) turned toward the source of the unexpected unilateral touch and raised their posture and major claws to confront it. A touch ispilateral to the recorded leg evoked vigorous depressor muscle activity that began immediately after the touch as the leg was depressed (Fig. 2B). A contralateral touch evoked a brief bout of activity before being silenced as the leg was raised and promoted for the turn (Fig. 2A). Robust depressor activity then occurred as the leg depressed during the first step.

These behavioral and neuromuscular responses to unilateral unexpected touch were highly consistent in socially isolated and dominant animals with electrodes implanted in the left fifth leg depressors (isolates $n=8$; dominants $n=8$; Fig. $3 A, B$ ). Unilateral touch stimuli were delivered to either side and evoked similar turning responses toward the touched side, regardless of which side was touched. Reflecting the asymmetry of this turning behavior, the depressors consistently produced larger responses to 

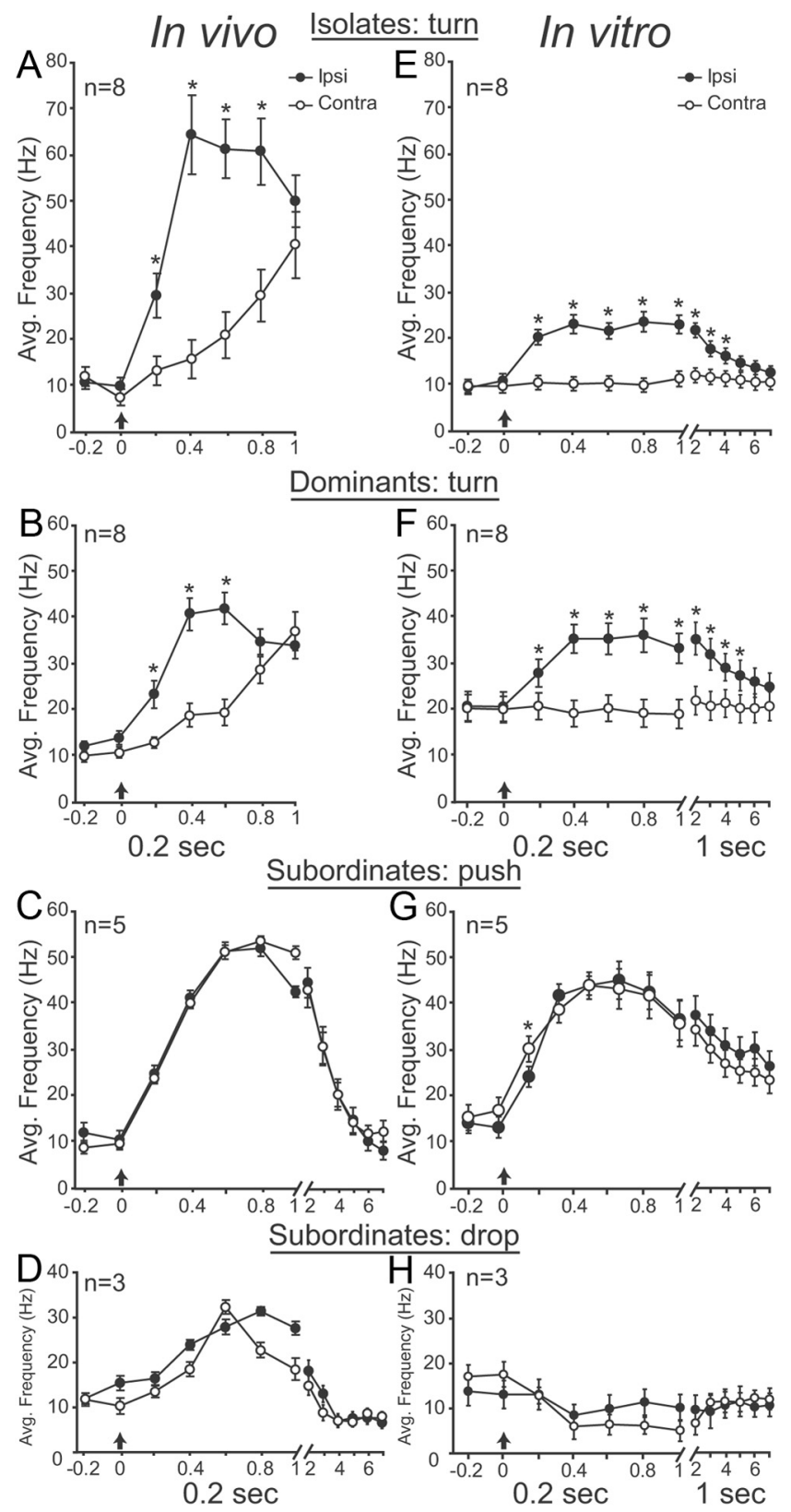

Figure 3. In vivo response of the depressor nerve and muscle EMG and in vitro response of the same depressor nerve to ipsilateral and contralateral sensory stimuli in socially isolated and experienced animals. $\boldsymbol{A}-\boldsymbol{D}$, Average ( \pm SEM) firing frequency responses of the depressor EMG units recorded in freely behaving isolates $(\boldsymbol{A})$, dominants $(\boldsymbol{B})$, and subordinates that push $(\boldsymbol{C})$ and drop $(\boldsymbol{D})$ during unexpected ipsilateral and contralateral touches (see Materials and Methods). $\boldsymbol{E}-\boldsymbol{H}$, Average ( \pm SEM) depressor nerve firing frequency responses recorded in in vitro preparations (Fig. $1 B$ ) of the same social isolates $(\boldsymbol{E})$, dominants $(\boldsymbol{F})$, and subordinates that pushed $(\boldsymbol{G})$ and dropped $(\boldsymbol{H})$ when tested in vivo. The in vitro responses shown $(\boldsymbol{E}-\boldsymbol{G})$ are from the depressornerve in the implanted leg of the in vivo preparations $(\boldsymbol{A}-\boldsymbol{D})$. Responses from the contralateral depressor nerve in each preparation were recorded simultaneously and had the same dependence on the side stimulated (data not shown). Arrows denote stimulus onset. The average firing frequencies during sequential $0.2 \mathrm{~s}$ periods were calculated for the response of each animal, and averages (and SEMs) of responses from corresponding periods were averaged across animals ( $n$ is given in each panel). Asterisks mark those times when the firing frequencies of the responses to ipsilateral and contralateral stimuli differed significantly according to a two-tailed Wilcoxon matched pairs test.

ipsilateral than to contralateral touch stimuli during the first $0.6 \mathrm{~s}$ following the stimulus.

Subordinates produced two different symmetric responses to the same unexpected unilateral touch: they either first pushed backward and then lowered their posture (push; Fig. 2C) or they flexed their abdomen and dropped their posture, and then moved backwards (drop; Fig. 2D). Bursts of depressor activity occurred initially during the push (Fig. $2 C$ ) or abdominal flexion (Fig. 2D) and were followed by no activity as the legs were raised to lower the animal's posture. Experiments on five subordinates that displayed the push response and three subordinates that displayed the drop response showed that for both types of animals, the depressor muscle responses to ipsilateral and contralateral stimuli were indistinguishable (Fig. 3C,D). However, the initial depressor responses during the push behaviors were nearly twice as large as during the drop behaviors. The status-related differences in the behavioral and muscular responses to unexpected unilateral touch were consistent across animals (Fig. $3 A-D)$.

The day after each in vivo experiment, the isolate, dominant, or subordinate animal was killed and a section of the animal's ventral nerve cord between the T2 and second abdominal (A2) ganglion was removed to a saline-filled dish together with both fifth walking legs (Fig. $1 B$ ). With this in vitro preparation, the first abdominal ganglion second nerve (A1 N2) that contained the axons of fringe hair afferents was stimulated electrically to evoke responses from depressor motor neurons in the T5 depressor nerve (Fig. 1). The depressor responses to unilateral A1 N2 stimulation retained their dependence on the animal's social status despite the absence of descending influences from the anterior CNS, including the brain (Fig. 3). In preparations from socially isolated and dominant crayfish, the responses of the left and right T5 leg depressor nerves depended on the side on which the A1 N2 was stimulated (Fig. $3 E, F$ ). As in the in vivo experiments (Fig. $3 A, B)$, the firing frequency in each depressor nerve significantly increased in response to ipsilateral A1 N2 stimulation, but showed little or no response to contralateral A1 N2 stimulation. The difference in the responses of the depressor nerve to ipsilateral and contralateral A1 N2 stimulation was statistically significant (two-tailed Wilcoxon matched pairs test, $p<0.0001$; isolates $n=8$, dominants $n=8$; Fig. $3 E, F)$.

The T5 depressor nerve recordings of in vitro preparations from subordinate animals showed two different but symmetric response patterns depending on whether the intact animal had displayed a push (Fig. 2C) or drop (Fig. 2D) response. The push in vitro responses (Fig. $3 G$ ) were excitatory and similar to the corresponding in vivo responses (Fig. $3 C$ ), whereas the drop in vitro responses were weakly inhibitory while the corresponding in vivo responses were weakly excitatory (Fig. $3 D, H$ ). Like the in vivo responses, the in vitro responses to stimuli of each side were similar: the difference in the firing frequencies of the depressor nerve to ipsilateral and contralateral A1 N2 stimulation was not significant (two-tailed Wilcoxon matched pairs test, $p>0.05$; subordinate push $n=5$, subordinates drop $n=3$ ).

The depressor nerve responses of 20 additional isolates, 13 dominants, and four subordinates were recorded in vitro but not in vivo and displayed the same status-dependent pattern (data not shown).

A bilaterally symmetric pair of anteriorly projecting, serotonin-containing neurons in the first abdominal ganglion, the A1 5-HT cells (Beltz and Kravitz, 1983; Real and Czternasty, 1990; Rossi-Durand, 1993; Heinrich et al., 1999), participates in the touch response. The A1 5-HT were identified from their shapes, discharge patterns, and immunoreactivity to serotonin antibody (see Methods and Materials, above). Their responses to unilateral A1 sensory stimulation displayed the same dependence on social status (Fig. 4) as the depressor motor neurons (Fig. 3). 

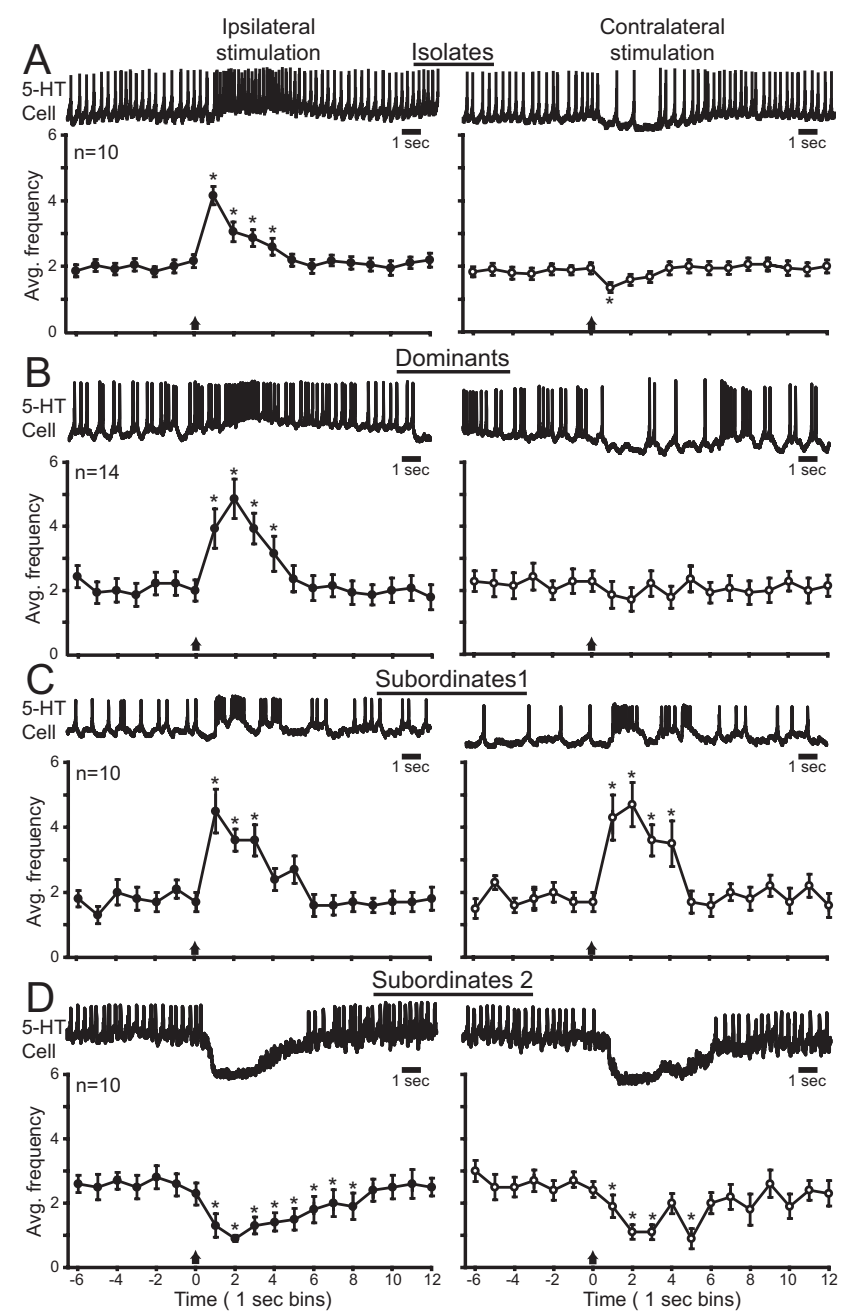

Figure 4. $\quad \boldsymbol{A}-\boldsymbol{D}$, Responses of the $\mathrm{A} 15-\mathrm{HT}$ cell to sensory stimulation in semi-intact preparations of social isolates $(\boldsymbol{A})$, dominants $(\boldsymbol{B})$, or subordinates $(\boldsymbol{C}, \boldsymbol{D})$. The recording at the top of each panel shows the intracellular response of the $\mathrm{A} 15$-HT cell to ipsilateral (left) and contralateral (right) touches of the lateral fringe hairs on the sides of the first abdominal segment. Averaged firing frequency responses for the set of animals tested are plotted below each trace. Arrows mark the time of the touch (time 0 ). Each point and error bar represents the average and SEM, respectively, across animals of corresponding firing frequencies averaged over $1 \mathrm{~s}$ periods before and after the stimulus (time 0 ). The asterisks indicate periods when the average frequency differed significantly from the averaged response at time 0 (statistical test for all conditions: two-tailed Wilcoxon matched pairs test, ${ }^{*} p<0.05$ ).

The cells fire tonically at rates that differ between preparations from $0.5-3 \mathrm{~Hz}$ to $15 \mathrm{~Hz}$ (Heinrich et al., 1999). In semi-intact preparations from socially isolated and dominant animals (see Materials and Methods, above; Fig. 1C), a brush stimulus applied to the fringe hairs of the first abdominal segment ipsilateral to a recorded A1 5-HT cell increased the cell's firing frequency, while the same brush stimulus applied contralaterally evoked either no response or modest inhibition (Fig. 4A,B). Responses of the 5-HT cell to ipsilateral and contralateral stimulation were measured as the difference between the peak (maximal or minimal) firing frequency and the frequency at the time of the stimulus. The difference between the 5-HT cell responses to ipsilateral and contralateral stimulation was statistically significant for both isolated and dominant animals (two-tailed Wilcoxon signed rank test, isolates $n=10, p=0.0039$; dominants $n=14, p=0.0001$ ).

In subordinate animals, brush stimuli applied to either side evoked similar A1 5-HT cell responses. In half of the subordinates tested, the A1 5-HT cell was similarly excited by ipsilateral and contralateral stimulation (Fig. 4C), while the cells in the remaining subordinates were similarly inhibited (Fig. 4D). For both groups, the difference in the responses of the 5-HT cell to ipsilateral and contralateral stimuli was not statistically significant (two-tailed Wilcoxon matched pairs tests, $p>0.05$; subordinates bi-excitation $n=10$; subordinates bi-inhibition $n=10$ ).

Like the depressor motor neurons, the 5-HT cells produced asymmetric response patterns in dominant and isolate animals, and symmetric patterns in subordinates. To test whether these patterns were different, we compared the differences in the animals' responses to ipsilateral and contralateral inputs. We found that the difference between the responses to ipsilateral and contralateral inputs in dominants differed significantly from the corresponding differences in both types of subordinates; a similar difference occurred between the responses of isolates and those of both types of subordinates (ANOVA with Tukey's multiple postcomparison test, $p<0.001)$. Conversely, the response differences of dominants were not different from those of isolates (ANOVA with Tukey's multiple postcomparison test, $p>0.05$ ).

The results presented above show that the depressor motor neurons and A1 5-HT interneurons on each side of the abdomen can produce responses to the same unilateral sensory stimulus (or sensory nerve stimulus) that depend on the social status and experience of the animal. In isolate and dominant animals, a unilateral stimulus excited an asymmetric pattern of responses in which the ipsilateral neurons were more strongly excited than the contralateral neurons, which might produce no response or be inhibited (Figs. $3 A, B ; 4 A, B$ ). In subordinate animals, two symmetric responses were recorded, either strong bilateral excitation (Figs. 3C, 4C) or weak bilateral excitation or inhibition (Figs. 3D, $4 D$ ). We have found that a simple model neural circuit composed of integrate-and-fire model neurons can account for these differences in the responses to unilateral sensory stimuli in isolate, dominant, and subordinate crayfish (see Materials and Methods, above; Fig. 5A). The set of depressor motor neurons on each side and the ipsilateral A1 5-HT interneuron are each represented by a model neuron. Single-model neurons also represent the set of sensory neurons on each side of the animal. Unilateral sensory stimulation is simulated by current injection into the corresponding model sensory neuron. Each sensory neuron excites a unilateral excitatory interneuron and bilateral excitatory and inhibitory interneurons. The unilateral excitatory interneuron excites the depressor motor neuron and 5-HT interneuron on the same side, whereas the excitatory and inhibitory bilateral interneurons excite and inhibit, respectively, the depressor motor neurons and 5-HT interneurons on both sides. Synaptic excitation and inhibition are mediated by changes in postsynaptic conductance that are coupled to depolarizing and hyperpolarizing reversal potentials, respectively (see Materials and Methods, above). The pattern of model neuron properties and synaptic contacts is symmetric about the midline.

Differences in the response of the network to unilateral sensory neuron stimulation were produced by changes in the relative thresholds of the circuit interneurons. An asymmetric response (Fig. 5B), similar to that seen in isolate and dominant animals, occurred when the threshold of the ipsilateral excitatory interneuron was lower than the threshold of the bilateral inhibitory interneuron, which was itself lower than that of the bilateral excitatory interneuron (Fig. 5C). With this pattern of thresholds, stimulation of one sensory neuron excited the ipsilateral depressor motor neuron and 5-HT interneuron and inhibited the contralateral cells. Here ipsilateral excitation was sufficiently strong 
A

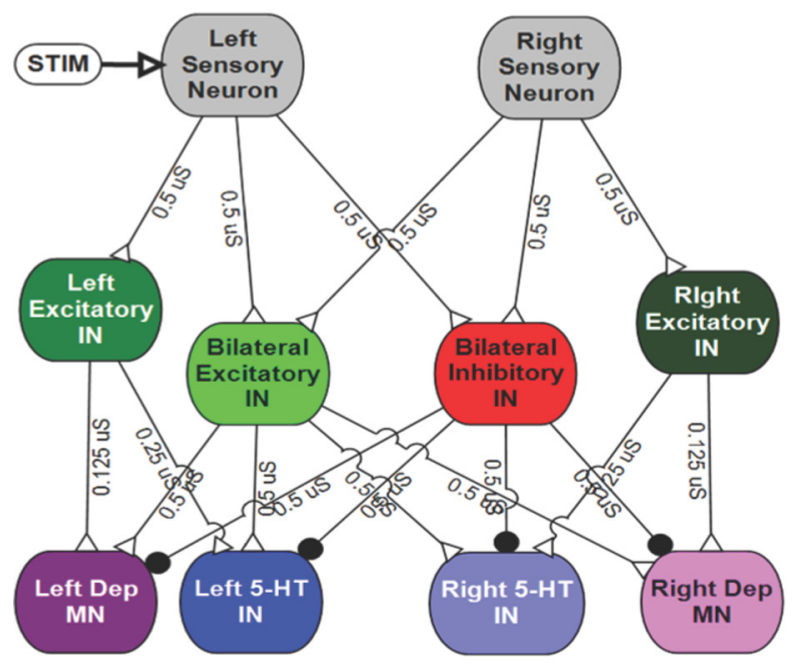

B

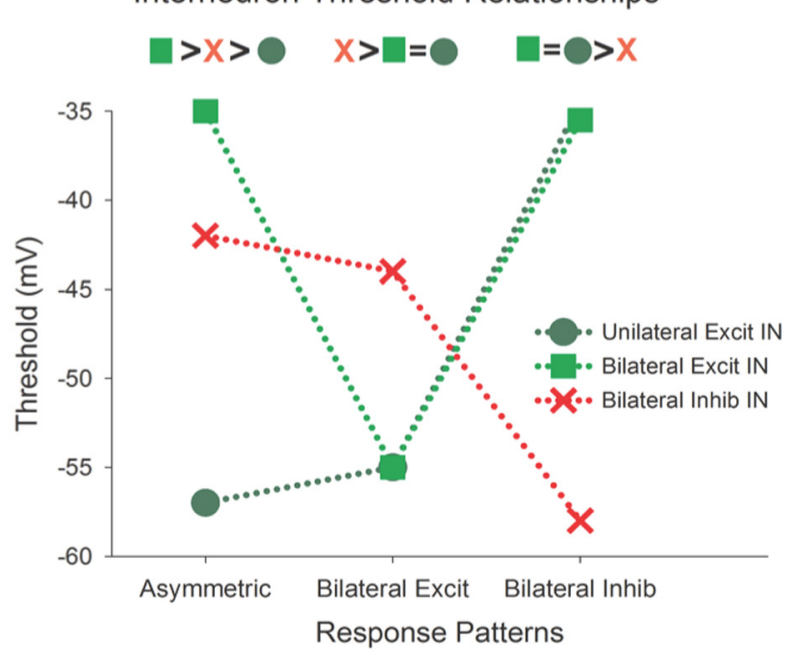

C

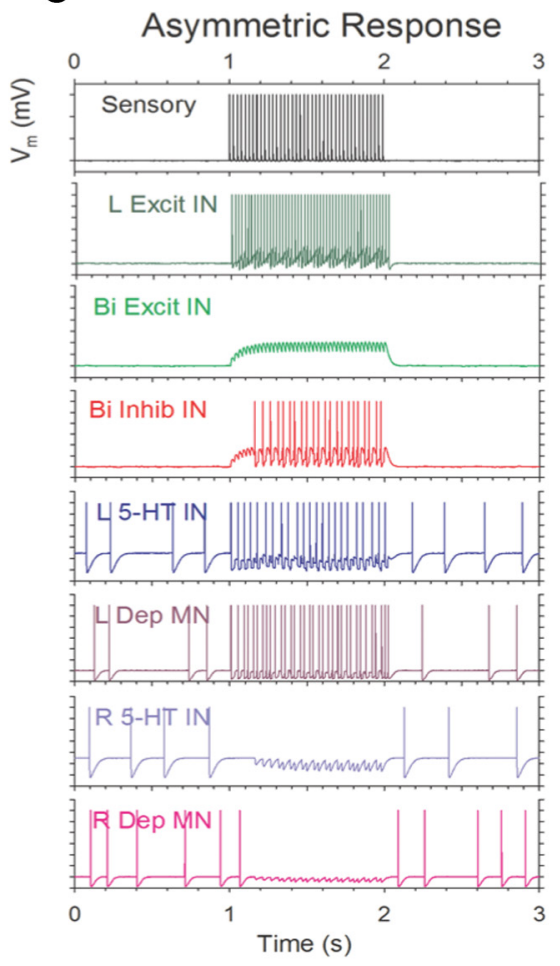

D

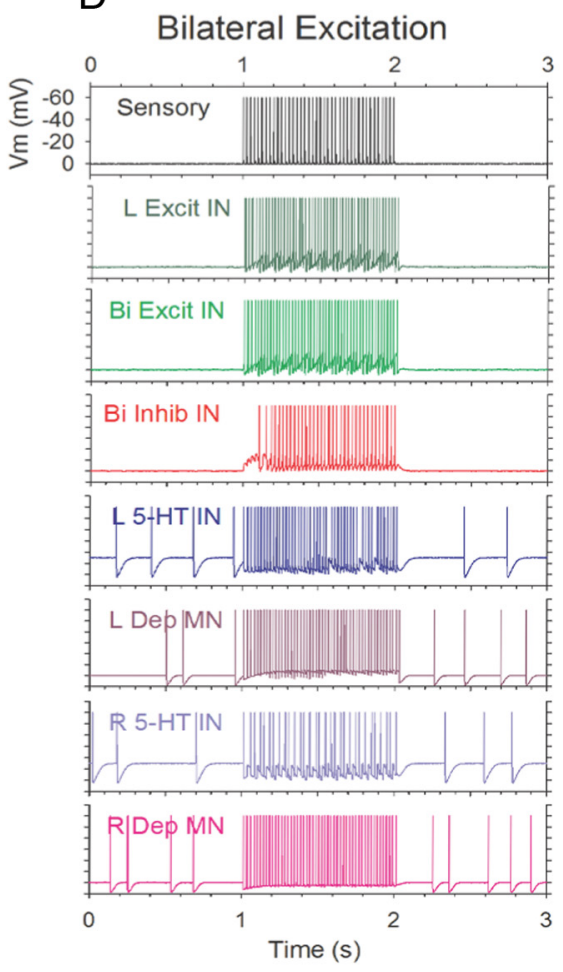

E

\section{Bilateral Inhibition}
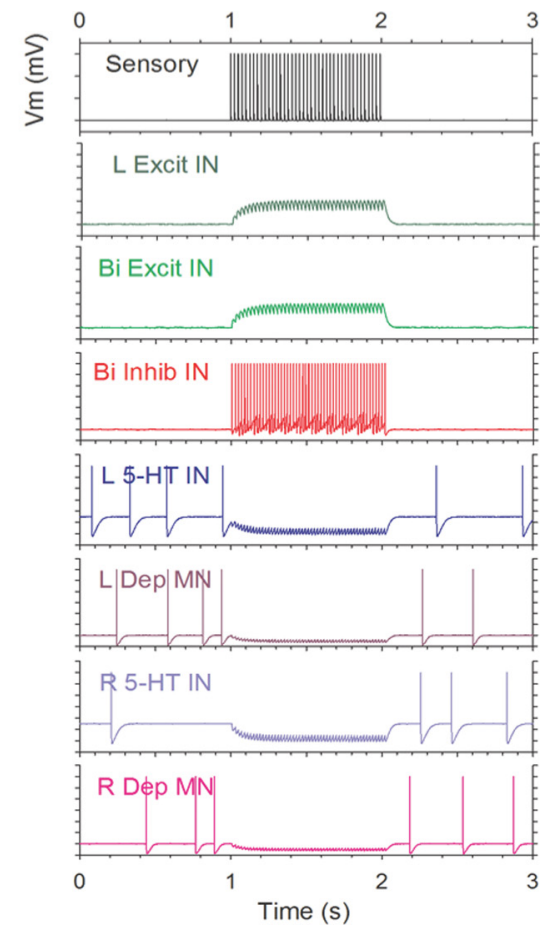

Figure 5. A model neural network that produces an asymmetric response typical of dominant and isolate animals and symmetric excited and inhibited responses typical of subordinate animals in response to unilateral stimulation. $\boldsymbol{A}$, Circuit diagram showing left and right integrate-and-fire sensory neurons (top), excitatory and inhibitory interneurons (IN; middle), and left and right depressor motor neurons (Dep MN) and 5-HT interneurons (bottom). The sensory stimulus (STIM) was simulated by a current injection to the left sensory neuron. $\boldsymbol{B}$, Three patterns of interneuron thresholds that produce the three types of responses (see text). $\boldsymbol{C}-\boldsymbol{E}$, Asymmetric, bilaterally excited (Bi Excit), and bilaterally inhibited (Bi Inhib) responses of the network to a unilateral sensory stimulus when the threshold relationships are those shown in $\boldsymbol{B}$. Trace colors correspond to model cell colors in $\boldsymbol{A}$.

to overcome the bilateral inhibition, but the bilateral excitation was not, so that ipsilateral excitation and contralateral inhibition resulted. When the thresholds of both the ipsilateral and bilateral excitatory interneurons were identical and lower (or even two millivolts higher) than that of the bilateral inhibitory interneuron, unilateral sensory neuron stimulation bilaterally excited the depressor motor neurons and 5-HT interneurons (Fig. 5B,D). When the thresholds were reversed and the thresholds of the excitatory interneurons were made higher than the inhibitory interneuron threshold, unilateral sensory neuron stimulation bi- laterally inhibited the depressors and 5-HT interneurons (Fig. $5 B, E)$. It is apparent that the three patterns of response result from the different relative balances of ipsilateral excitation, bilateral excitation, and bilateral inhibition in this network.

\section{Discussion}

Dominant and subordinate animals are recognizable from characteristic differences in their behavioral responses to the same sensory stimuli. When touched on the side by an unexpected stimulus, a dominant or a socially isolated crayfish will usually 
turn in place toward the side of the touch and raise its claws to confront the stimulus source (Song et al., 2006). Socially subordinate crayfish will withdraw backwards or drop in place and then withdraw in response to the same unilateral stimulus. Here we have shown that those differences are apparent in the responses of the leg depressor motor neurons in intact animals, and that the different motor neuronal responses persist when the thoracic ganglia containing them are removed from the animal. Moreover, these response differences extend to the pair of serotonergic interneurons, the A1 5-HT cells, that project anteriorly to all five thoracic ganglia that govern movements of the four pairs of walking legs and the claws (Real and Czternasty, 1990).

Both the dominant and isolate crayfish turned toward the side of the touch, depressing the ipsilateral leg by exciting the ipsilateral depressors, and lifting the contralateral leg, presumably by exciting the contralateral levators. The asymmetric responses of the depressor motor neurons seen in both the intact animal and in vitro preparation of the same animal are appropriate to mediate the turning response. Subordinate animals display symmetric withdrawals in response to the unilateral stimulus that are mediated either by bilateral excitation of the depressors (the push response of Fig. 2C) or bilateral inhibition (the drop response of Fig. 2D).

The results from isolated nerve cords show that the statusrelated response differences are mediated by changes in local excitatory and inhibitory pathways that link primary sensory afferents to the depressor motor neurons and A1 5-HT interneurons. Earlier results suggested that status-mediated changes in these pathways are likely to include changes in the depressor motor neurons themselves: both the levels of spontaneous activity of the leg depressor and levator motor neurons and the amplitudes of their resistance reflexes to proprioceptive inputs are higher in dominant than in subordinate animals (Cattaert et al., 2010). In addition, exposure of the isolated ganglion to serotonin increased the differences in resistance reflexes seen in depressor motor neurons while increasing the electrical input resistance in dominant animals and decreasing it in subordinates.

Our simulations show that differences in the relative thresholds of excitatory and inhibitory interneurons in a simple sensory-motor circuit can account for the status-dependent response differences of depressor motor neurons and 5-HT interneurons described here. In this circuit, the output neurons on both sides of the animal are affected by unilateral sensory stimuli through pathways that mediate ipsilateral excitation, bilateral excitation, and bilateral inhibition. Differences in the thresholds of elements in these pathways produce responses characteristic of a particular status. However, relative differences in other parameters of the pathways, such as synaptic efficacy or input resistance of the neurons, could produce similar effects, leading to the conclusion that the status-dependent effects appear to result from differences in the relative excitabilities of circuit pathways that produce ipsilateral excitation, bilateral excitation, and bilateral inhibition (Calin-Jageman et al., 2007; Issa et al., 2011; Koch et al., 2011).

Serotonin modulates the responsiveness of depressor motor neurons according to the social status of the animal (Cattaert et al., 2010). This result and that of Figure 4 suggest that the A1 5-HT neurons' responsiveness and the effects of the serotonin they release may contribute synergistically to the status dependence of the depressor motor neuron responses. Whatever signals produce the modulatory changes associated with social status can be seen to affect serotonergic modulation of neuronal circuits, as well as the circuits themselves. This metamodulation, which is also seen in the effect of status on serotonergic modula- tion of escape (Edwards et al., 2002), is a general phenomenon (Katz and Edwards, 1999) that affects the behavioral states and hormonal function in a variety of animals (Fernald, 2002; Robinson et al., 2008; O'Connell and Hofmann, 2011).

The internal signals that mark a change in social status are not known for any animal, but in crayfish they lead to neural circuit reconfigurations that are retained locally in parts of the CNS after their separation from the brain and the body. Like the statusdependent modulatory effects of 5-HT on the command neurons for escape (Yeh et al., 1996, 1997), the thoracic circuit configuration persists for hours after removal from the body and so does not depend on a continuous status-related neural or hormonal signal from the brain. Earlier work has suggested that a hormonal signal was sufficient both to induce and maintain a social-statusrelated change in the excitability of abdominal escape circuits that occurred after the connection to higher centers was cut (Arfai and Krasne, 1999). A similar experiment might reveal whether such a signal can also induce the persistent changes in thoracic reflexes reported here. If so, the sources of these signals might be sought in the neuroendocrine structures of the eyestalks (Khalaila et al., 2002).

The many behavioral differences of dominant and subordinate crayfish suggest that status-related differences in neural function extend to circuits throughout the crayfish nervous system. For example, the excitability of both medial giant and nongiant escape circuitry is much higher in subordinate than in dominant crayfish during their agonistic interactions (Herberholz et al., 2001), whereas the excitability of the lateral giant (LG) escape circuitry is lower in subordinates than in dominants (Krasne et al., 1997). The cellular and synaptic changes that underlie these differences are largely unknown, but appear to include differences in the effects of neuromodulators on synaptic excitation. For example, 5-HT has been shown to enhance the synaptic response of the LG command neuron for escape in dominants and inhibit it in subordinates (Yeh et al., 1996, 1997). These results indicated that a change in status creates a persistent change in distant parts of the nervous system by mechanisms that may include changes in the type or balance of 5-HT receptors expressed in target cells (Spitzer et al., 2005, 2008).

The variety and similarity of differences in the behavior of dominant and subordinate animals of many social species suggests that neural circuit reconfiguration that follows a change in social status is widespread among higher animals. For example, the excitabilities of the Mauthner neuron in fish and the startle response that the neuron triggers both depend on the social status of the animal (Neumeister et al., 2010). Status-dependent circuit reconfiguration in other species may result from proximate mechanisms similar to those described in crayfish, including changes in modulatory function, neuronal thresholds, and the gain or sign of synaptic function (Yeh et al., 1996; Krasne et al., 1997; Herberholz et al., 2001). Indeed, as in crayfish (Yeh et al., 1996; Cattaert et al., 2010), the status-dependent changes in Mauthner excitability have been linked to status-dependent changes in serotonergic modulation (Whitaker et al., 2011). Other proximate mechanisms may result from the kinds of status-related differences in the nervous system already observed in many animals, including transmitter or neuromodulator concentration (Gutzler et al., 2010), receptor populations (Spitzer et al., 2005; Burmeister et al., 2007), changes in neuronal size and shape (White et al., 2002), neurogenesis (Kozorovitskiy and Gould, 2004; Song et al., 2007), and gross brain morphology (Holmes et al., 2007; O’Donnell et al., 2007). 


\section{References}

Antonsen BL, Edwards DH (2003) Differential dye coupling reveals lateral giant escape circuit in crayfish. J Comp Neurol 466:1-13.

Arfai N, Krasne FB (1999) Social experience-dependent changes in response of crayfish lateral giant neurons to 5 -HT may be induced by humoral factors. Soc Neurosci Abstr 25:69.13.

Beltz BS, Kravitz EA (1983) Mapping of serotonin-like immunoreactivity in the lobster nervous system. J Neurosci 3:585-602.

Burmeister SS, Kailasanath V, Fernald RD (2007) Social dominance regulates androgen and estrogen receptor gene expression. Horm Behav 51:164-170.

Calin-Jageman RJ, Tunstall MJ, Mensh BD, Katz PS, Frost WN (2007) Parameter space analysis suggests multi-site plasticity contributes to motor pattern initiation in Tritonia. J Neurophysiol 98:2382-2398.

Cattaert D, Delbecque JP, Edwards DH, Issa FA (2010) Social interactions determine postural network sensitivity to 5-HT. J Neurosci 30:5603-5616.

Copp NH (1986) Dominance hierarchies in the crayfish Procambarus clarkii (Girard, 1852) and the question of learned individual recognition (Decapoda, Atacidea). Crustaceana 51:9-23.

Edwards DH, Yeh SR, Musolf BE, Antonsen BL, Krasne FB (2002) Metamodulation of the crayfish escape circuit. Brain Behav Evol 60:360-369.

Edwards DH, Issa FA, Herberholz J (2003) The neural basis of dominance hierarchy formation in crayfish. Microsc Res Tech 60:369-376.

Fernald RD (2002) Social regulation of the brain: sex, size and status. Novartis Found Symp 244:169-184.

Gutzler SJ, Karom M, Erwin WD, Albers HE (2010) Arginine-vasopressin and the regulation of aggression in female Syrian hamsters (Mesocricetus auratus). Eur J Neurosci 31:1655-1663.

Heinrich R, Cromarty SI, Hörner M, Edwards DH, Kravitz EA (1999) Autoinhibition of serotonin cells: an intrinsic regulatory mechanism sensitive to the pattern of usage of the cells. Proc Natl Acad Sci U S A 96:2473-2478.

Herberholz J, Issa FA, Edwards DH (2001) Patterns of neural circuit activation and behavior during dominance hierarchy formation in freely behaving crayfish. J Neurosci 21:2759-2767.

Herberholz J, McCurdy C, Edwards DH (2007) Direct benefits of social dominance in juvenile crayfish. Biol Bull 213:21-27.

Holmes MM, Rosen GJ, Jordan CL, de Vries GJ, Goldman BD, Forger NG (2007) Social control of brain morphology in a eusocial mammal. Proc Natl Acad Sci U S A 104:10548-10552.

Issa FA, Adamson DJ, Edwards DH (1999) Dominance hierarchy formation in juvenile crayfish, Procambarus clarkii. J Exp Biol 202:3497-3506.

Issa FA, O’Brien G, Kettunen P, Sagasti A, Glanzman DL, Papazian DM (2011) Neural circuit activity in freely behaving zebrafish (Danio rerio). J Exp Biol 214:1028-1038.

Katz PS, Edwards DH (1999) Metamodulation: the control and modulation of neuromodulation. In: Neuromodulation: its role in information flow and neuronal circuit flexibility (Katz PS, ed), Oxford: Oxford UP.

Khalaila I, Manor R, Weil S, Granot Y, Keller R, Sagi A (2002) The eyestalk- androgenic gland-testis endocrine axis in the crayfish Cherax quadricarinatus. Gen Comp Endocrinol 127:147-156.

Koch H, Garcia AJ 3rd, Ramirez JM (2011) Network reconfiguration and neuronal plasticity in rhythm-generating networks. Integr Comp Biol 51:856-868.

Kozorovitskiy Y, Gould E (2004) Dominance hierarchy influences adult neurogenesis in the dentate gyrus. J Neurosci 24:6755-6759.

Krasne FB, Shamsian A, Kulkarni R (1997) Altered excitability of the crayfish lateral giant escape reflex during agonistic encounters. J Neurosci 17:709-716.

Neumeister H, Whitaker KW, Hofmann HA, Preuss T (2010) Social and ecological regulation of a decision-making circuit. J Neurophysiol 104:3180-3188

O'Connell LA, Hofmann HA (2011) Genes, hormones, and circuits: an integrative approach to study the evolution of social behavior. Front Neuroendocrinol 32:320-335.

O’Donnell S, Donlan N, Jones T (2007) Developmental and dominanceassociated differences in mushroom body structure in the paper wasp Mischocyttarus mastigophorus. Dev Neurobiol 67:39-46.

Real D, Czternasty G (1990) Mapping of serotonin-like immunoreactivity in the ventral nerve cord of crayfish. Brain Res 521:203-212.

Robinson GE, Fernald RD, Clayton DF (2008) Genes and social behavior. Science 322:896-900.

Rossi-Durand C (1993) Peripheral proprioceptive modulation in crayfish walking leg by serotonin. Brain Res 632:1-15.

Song CK, Herberholz J, Edwards DH (2006) The effects of social experience on the behavioural response to unexpected touch in crayfish. J Exp Biol 209:1355-1363.

Song CK, Johnstone LM, Schmidt M, Derby CD, Edwards DH (2007) Social domination increases neuronal survival in the brain of juvenile crayfish Procambarus clarkii. J Exp Biol 210:1311-1324.

Spitzer N, Antonsen BL, Edwards DH (2005) Immunocytochemical mapping and quantification of expression of a putative type 1 serotonin receptor in the crayfish nervous system. J Comp Neurol 484:261-282.

Spitzer N, Edwards DH, Baro DJ (2008) Conservation of structure, signaling and pharmacology between two serotonin receptor subtypes from decapod crustaceans, Panulirus interruptus and Procambarus clarkii. J Exp Biol 211:92-105.

Whitaker KW, Neumeister H, Huffman LS, Kidd CE, Preuss T, Hofmann HA (2011) Serotonergic modulation of startle-escape plasticity in an African cichlid fish: a single-cell molecular and physiological analysis of a vital neural circuit. J Neurophysiol 106:127-137.

White SA, Nguyen T, Fernald RD (2002) Social regulation of gonadotropinreleasing hormone. J Exp Biol 205:2567-2581.

Wilson EO (1975) Sociobiology. Cambridge, MA: Harvard UP.

Yeh SR, Fricke RA, Edwards DH (1996) The effect of social experience on serotonergic modulation of the escape circuit of crayfish. Science 271:366-369.

Yeh SR, Musolf BE, Edwards DH (1997) Neuronal adaptations to changes in the social dominance status of crayfish. J Neurosci 17:697-708. 